\title{
Quelles contributions des élites « rouges » au façonnement des États post-coloniaux?
}

Patrice Yengo et Monique de Saint Martin

\section{OpenEdition}

1 Journals

Édition électronique

URL : http://journals.openedition.org/etudesafricaines/20661

DOI : 10.4000/etudesafricaines.20661

ISSN : 1777-5353

Éditeur

Éditions de l'EHESS

\section{Édition imprimée}

Date de publication : 1 juillet 2017

Pagination : 231-258

ISBN : 978-2-7132-2688-5

ISSN : 0008-0055

\section{Référence électronique}

Patrice Yengo et Monique de Saint Martin, "Quelles contributions des élites « rouges » au façonnement des États post-coloniaux? », Cahiers d'études africaines [En ligne], 226 | 2017, mis en ligne le 01 avril 2017, consulté le 23 septembre 2020. URL : http://journals.openedition.org/ etudesafricaines/20661 ; DOI : https://doi.org/10.4000/etudesafricaines.20661 


\section{Quelles contributions des élites « rouges » au façonnement des États post-coloniaux?}

La question de l'État en Afrique, de sa formation, de sa nature ou de son évolution, est encore loin d'être épuisée. Qu'elles soient restées au stade du néocolonialisme ou qu'elles se revendiquent d'une autonomie assumée, les études qui la soulèvent s'accordent toutes au moins à reconnaître que sa conception est à caractère tératologique ${ }^{1}$, qu'il s'agisse de l'État comme organisation politique définie uniquement par le périmètre géographique tracé par la colonisation, ou de l'État comme nation où le regroupement des différentes composantes est pour une large part le fruit des tribulations conflictuelles des impérialismes du XIX ${ }^{\mathrm{e}}$ siècle. Pourtant, c'est dans l'héritage de ce dérèglement initial que les États africains ont été obligés de trouver une voie pour construire leur souveraineté prise dans la vision évolutionniste du monde en vigueur et bordée par les impératifs du développement économique. Structurer un appareil d'État susceptible de répondre aux exigences de ce développement, telle était la tâche immédiate que s'étaient fixés les États africains au lendemain des Indépendances en 1960.

À cet effet, dès 1960 et durant la Guerre froide, de très nombreux étudiants venus de la quasi-totalité des pays de l'Afrique indépendante — se réclamant ou non du socialisme - sont partis en URSS, et dans les autres pays du bloc de l'Est ainsi qu'à Cuba et parfois en Chine, pour y suivre une formation. Confrontés à la pénurie de cadres au lendemain des Indépendances, les nouveaux États africains, dont les étudiants étaient jusque-là préférentiellement formés dans les pays colonisateurs, se sont alors tournés aussi vers l'URSS et les pays de l'ex-bloc socialiste pour accélérer la formation d'une partie importante de leurs cadres.

Loin d'être les sujets passifs de l'impérialisme des pays occidentaux ou de l'URSS, les pays africains jouaient alors sur plusieurs registres de la compétition entre les deux blocs ${ }^{2}$, et multipliaient, autant qu'ils le pouvaient, les

1. Voir notamment J.-F. BAYART (1989).

2. Si le terme «bloc» est ici utilisé, c'est pour désigner les mondes qui s'affrontaient en particulier pendant la période de la Guerre froide. Cependant, ce qui 
interlocuteurs en envoyant au même moment leurs étudiants dans différentes directions. Pour la plupart des États du Sud, la coopération avec les pays de l'ancien bloc socialiste relevait avant tout d'un choix pragmatique dicté par une volonté profonde d'indépendance politique qui en était aussi le socle idéologique. C'est à l'aune du projet de l'indépendance qu'il faut comprendre les motivations des mouvements de libération des pays encore sous tutelle coloniale : Mouvement populaire de libération de l'Angola (MPLA), Parti africain pour l'indépendance de la Guinée et du Cap-Vert (PAIGC), Congrès national africain (ANC), ou des partis clandestins : Front national de libération du Mozambique (Frelimo), Organisation du peuple de l'Afrique du Sud-Ouest (SWAPO), Union nationale africaine du Zimbabwe (ZANU) ${ }^{3}$ qui ont envoyé leurs militants se former dans les pays du bloc socialiste, prioritairement dans les métiers de la guerre, mais aussi dans ceux relevant de la gestion du futur État.

Partir dans les pays du bloc socialiste, une approche oblique

Après un premier bilan de cette coopération éducative inédite aux formes multiples - soutien aux mouvements de libération, partenariat formalisé entre États - entre nouveaux États africains et pays socialistes ainsi que de son impact sur le devenir de nouvelles élites africaines réalisé par Constantin Katsakioris, ce numéro, qui explore principalement le retour de ces élites après leurs études, prend en compte des expériences diverses comme le régime du Derg et ses élites aujourd'hui complètement discrédités en Éthiopie ou le Parti congolais du travail et ses démêlés avec le mouvement étudiant en URSS. Il focalise ensuite sur des situations aux marges des processus assez massifs de départ, de formation dans les pays de l'ex-bloc socialiste, et de retour dans le pays d'origine, tels la coopération éducative au féminin pour les Maliennes, le devenir d'étudiants touaregs partagés à leur retour entre la République du Mali et l'Azawad, le déclassement d'étudiants réunionnais en quête d'indépendance pour leur île, la mise en place d'un art prolétarien «à la congolaise ». Celles-ci sont susceptibles d'éclairer la façon selon laquelle se sont opérées la formation et la circulation de cadres experts et « rouges ».

Une attention forte est prêtée au terrain, aux circulations d'acteurs, de savoirs, de pratiques, de modèles, et de représentations entre URSS, autres pays de l'ex-bloc socialiste et Afrique, ainsi qu'aux interactions Est/Sud;

nous intéresse ici, ce sont moins les luttes et les rivalités entre l'Est et l'Ouest que les complexités de chacun de ces mondes, les interconnexions, les échanges entre divers espaces nationaux. Sur ce point voir Boulland \& Gouarné (2015).

3. Passant assez souvent par des pays relais pour venir, les étudiants africains étaient les plus nombreux en URSS parmi les étudiants étrangers, plus même que les étudiants asiatiques. Des élèves du secondaire, par exemple à Cuba ou en RFA, des stagiaires pour des formations courtes ou encore des militaires ont aussi été envoyés dans les pays socialistes. 
on est ainsi loin d'une approche ou d'un «programme surplombant», pour reprendre l'expression de Luc Boltanski ${ }^{4}$. C'est plutôt une approche ou un regard en oblique qui, pour essayer de déchiffrer un processus ou un phénomène, s'en détourne, comme le proposait récemment dans un tout autre contexte Carlo Ginzburg 5 . Deux cas illustrent cette approche.

Seules une centaine de femmes maliennes ont pu se rendre en URSS entre 1961 et 1991 dans le cadre de la coopération entre le Mali et l'URSs et avec des bourses du Comité des femmes soviétiques ; elles sont devenues puéricultrices, jardinières d'enfants, assistantes sociales, sages-femmes, plus rarement médecins, et n'ont pas toujours été des militantes. Cependant, comme l'analysent Tatiana Smirnova et Ophélie Rillon, quelques-unes sont parvenues à transgresser les frontières de genre pourtant fortement affirmées, voire à ébranler l'hégémonie masculine de l'appareil d'État. Même si les militantes africaines et soviétiques n'avaient pas toujours les mêmes visions et représentations de l'émancipation des femmes et des combats à mener, si les tensions étaient souvent fortes, elles ont tenté de façonner ensemble une solidarité féministe internationale, et y ont pour une part réussi, parfois en s'appuyant sur des liens personnels ${ }^{6}$. Ce qui est particulièrement frappant, c'est que contre toute attente, cette coopération féminine éducative s'est prolongée et s'est accentuée après la chute du régime socialiste malien.

Le nombre d'étudiants touaregs formés dans l'ancien bloc de l'Est (URSS, Cuba, Tchécoslovaquie, Roumanie) dans les années 1960-1990 est très faible, une vingtaine environ, comparativement à celui des étudiants maliens ayant obtenu un diplôme dans les mêmes pays, environ 2500 pour la seule URSS entre 1962 et 1993 ; ils ont étudié pour la quasi-totalité d'entre eux dans des spécialités dites techniques. L'article d'Amalia Dragani n'en permet pas moins de comprendre la dimension internationale d'acteurs locaux, issus de minorités d'origine nomade et pastorale, en éclairant leurs choix politiques respectifs ainsi que leurs éventuels engagements dans les mouvements politiques générés par les soubresauts de l'État post-colonial.

\section{Une histoire de mobilités des étudiants et des élites}

Il est étonnant de constater que les différentes mobilités d'étudiants africains et asiatiques vers les pays de l'ancien bloc socialiste, qui ont concerné parfois des groupes restreints, mais le plus souvent des contingents importants,

4. Dans sa tentative pour rendre compatibles ou concilier ce qu'il appelle la « sociologie critique surplombante » et les sociologies pragmatiques de la critique (BOLTANSKI 2009).

5. «Pour comprendre le présent, nous devons apprendre à le regarder en oblique » (GINZBURG 2009 : 23).

6. Ceci n'était d'ailleurs sans doute pas une spécificité de la coopération féminine et pourrait s'observer de façon plus générale dans la coopération éducative URSS/ pays du Sud. 
et qui comptent parmi les plus fortes mobilités étudiantes du $\mathrm{XX}^{\mathrm{e}}$ siècle, sont restées longtemps oubliées des travaux sur les circulations universitaires et la structuration des États africains, alors que les migrations internationales d'étudiants en Europe (Karady 2002), les migrations Sud/Nord, ou les mobilités étudiantes dans les anciennes métropoles coloniales ont donné lieu à différents travaux, et que les mobilités Sud/Sud commencent elles aussi à être étudiées (Eyebiyi \& Mazzella 2014).

Les causes de cet oubli sont nombreuses. Peut-être, suggère Jean-Pierre Dozon (2015 : 12), « cela a-t-il à voir avec l'échec du système soviétique lui-même et avec celui des régimes socialistes ou marxistes-léninistes qui s'étaient mis en place en Afrique même, comme si il fallait laisser du temps pour assimiler ces échecs, prendre plus précisément la mesure par un examen distancié de tout ce que la période à laquelle ils ont mis fin a pu recéler, spécialement du côté africain, comme espérances, trajectoires et mémoires particulières ». À cela, il faut ajouter le discrédit porté sur ces élites dans de très nombreux pays d'Afrique ou encore l'absence d'associations d'anciens étudiants formés en URSS qui revendiqueraient la reconnaissance de leurs diplômes et développeraient un esprit de corps, au point que l'identité soviétique de leurs études est souvent camouflée ou passée sous silence comme le montre l'exemple de l'Éthiopie.

Sans entrer dans l'analyse de l'évolution des flux d'étudiants partis d'Afrique vers les pays de l'ancien bloc socialiste, un rappel de quelques données permet une appréciation de l'ampleur relative des mobilités des étudiants africains, en particulier durant la Guerre froide. De 1956 à 1991, environ 60000 étudiants de pays arabes ${ }^{7}$ et 56000 étudiants de pays d'Afrique subsaharienne se sont rendus pour étudier en URSS qui apparaît ainsi comme la principale destination dans l'ancien bloc socialiste ${ }^{8}$. Au moment de la dissolution de l'URSS en décembre 1991, on compte 47312 diplômés des pays arabes et 43500 des pays africains ${ }^{9}$, la majorité d'entre eux dans des universités, des écoles de médecine ou d'ingénieurs (Katsakioris 2016). Alors

7. Cette distinction entre «pays arabes » et «pays d'Afrique subsaharienne » est faite par les ministères et autres organismes recueillant les données statistiques en URSS/Russie. Dans le groupe de «pays arabes », sont inclus l'Algérie, le Maroc, la Tunisie, la Libye, l'Égypte, le Soudan, la Syrie, l'Irak, la Jordanie, le Liban, le Yémen du Nord, le Yémen du Sud, l'Oman, l'Arabie saoudite, le Qatar, le Bahreïn, le Koweït, les Émirats arabes unis et les territoires habités par les Palestiniens qui font partie de l'État palestinien.

8. Sans doute, les données statistiques actuellement disponibles sur les étudiants africains venus dans les pays de l'Est, ou sur la répartition des diplômés africains par pays d'origine, université, discipline, sexe, âge, année, etc. comportent-elles des lacunes, manquent-elles de précisions, et présentent-elles d'importantes divergences entre elles, en raison notamment de l'utilisation de variations dans les définitions de l'étudiant : "étudiants académiques » pour la CIA, «étudiants internationaux » de l'UNESCO et dans les procédures d'agrégation des effectifs (LECLERC-Olive \& BÉTHUNe 2013).

9. Les promotions de 1987 et 1988 qui ont eu leur diplôme dans un pays postsoviétique après décembre 1991 ne sont pas comptées. 
qu'en 1962, le nombre d'étudiants africains arrivant en URSS est trois fois inférieur à celui de ceux orientés vers la France, vers le Royaume-Uni ou vers les USA, à partir de 1979, les étudiants africains formés en URSS dépassent en nombre ceux allant étudier au Royaume Uni, et de 1988 à 1991 dépassent même ceux qui partent aux USA, la France constituant depuis 1984 le principal pays d'accueil des étudiants venant d'Afrique. Après la perestroüka, les effectifs d'étudiants de pays africains et arabes formés en Russie s'effondrent avant de remonter à partir de 1999.

La progression du nombre d'étudiants africains est un peu plus tardive dans les autres pays socialistes qui n'ont adopté ni la même politique d'accueil, ni le même rythme. Les étudiants africains sont au total un peu moins nombreux dans les démocraties populaires qu'en URSS. La RDA représente le pays accueillant le plus d'élèves et offrant les meilleures conditions d'accueil. Suivent la Roumanie ${ }^{10}$, la Pologne, la Tchécoslovaquie, la Hongrie, la Bulgarie.

En Chine, l'accueil des premiers étudiants africains remonte aux années 1950 selon la presse chinoise dépouillée par Antoine Kernen et Antoine Guex, à l'établissement de relations diplomatiques avec l'Égypte en 1956, mais c'est surtout après la création de l'Institut des langues étrangères de Pékin en octobre 1964, que le nombre d'étudiants africains dans les universités chinoises devient significatif ${ }^{11}$. «Depuis les années 1960 jusqu'aux réformes économiques engagées fin 1978, les boursiers africains sont mis en scène dans la propagande chinoise puisqu'ils incarnent l'engagement politique de la Chine maoïste à l'égard du "tiers-monde". Bien que hautement symbolique, le nombre des étudiants africains reste très limité. Ils ne sont que 836 à avoir obtenu une bourse durant les années 1970, et 2245 dans les années 1980, souvent présentées comme étant celles du désengagement chinois en Afrique » (Kernen \& Guex 2016 : 8). Ce nombre augmente fortement ensuite : entre 1999 et 2007 , le nombre d'étudiants africains en Chine a plus que quadruplé, passant de 1386 à 5 923, selon le ministère chinois de l'Éducation, mais il ne représentait que $3 \%$ de l'ensemble des étudiants étrangers (Bredeloup 2014). Les étudiants africains sont ensuite de plus en plus nombreux à s'installer en Chine après leurs études.

10. Sur les étudiants africains en Roumanie et en RDA, voir GHEORGHIU \& NETEDU (2015). Selon un des documents consultés par ces deux auteurs, en 1977 il y avait 4052 étudiants africains en Roumanie, sur un total de 11129 étudiants étrangers ; sur la RDA, voir aussi BolTOVSKAJA (2014). Des recherches ont également été entreprises sur les étudiants africains venus en Pologne, en République tchèque, en Bulgarie. Voir à ce sujet <http://www.fmsh.fr/fr/27007>, le programme de la conférence du programme ÉLITAF «Étudiants africains en URSS et dans les autres pays du monde communiste, 1960-1990. Entre histoires nationales et contexte international », Paris, 20-21 novembre 2014.

11. L'initiative de la création de cet institut est généralement attribuée à Zhou En Lai qui aurait, au lendemain de son voyage en Afrique, signifié la nécessité de renforcer les compétences linguistiques en Chine et de se donner les moyens d'accueillir des étudiants boursiers étrangers. 
La politique de coopération de Cuba avec les pays africains commence au lendemain de la Révolution; les élèves et étudiants africains y sont fort nombreux et restent souvent très longtemps (30 719 originaires de 42 pays africains différents entre 1961 et 2007 dont 17906 pour l'enseignement secondaire et 12813 dans l'enseignement supérieur) (González López 2008 ; Gómez Martin 2016). L'Isla de la Juventud en accueille un grand nombre dans ses écoles professionnelles et ses universités.

Les départs des anciens étudiants de pays d'Afrique formés dans les anciens pays socialistes, leurs expériences et leurs trajectoires dans leur complexité et leur diversité, mais aussi la politique d'accueil des étudiants africains par les pays socialistes — souvent modifiée et revue, mise en œuvre par ses principaux acteurs institutionnels, tel le Parti communiste de l'Union soviétique (PCUS), semi institutionnels, telles les amicales d'étudiants, et individuels - ont donné lieu à de premières analyses dans le cadre du programme ÉLITAF ${ }^{12}$ (de Saint Martin, Scarfò Ghellab \& Mellakh 2015). Des enquêtes précises et approfondies ont été réalisées auprès d'étudiants partis de différents pays de l'Afrique au nord du Sahara vers les pays de l'ancien bloc socialiste (Leclerc-Olive 2016). D'autres initiatives d'envergure sont en chantier dans plusieurs universités européennes. Ainsi, Miles Larmer et Paul Betts, dans le cadre du projet « Socialism Goes Global » lancé à l'Université d'Exeter ${ }^{13}$, ont entre autres organisé à Oxford une conférence «Africa, Eastern Europe and the Dream of International Socialism : New Perspectives on the Global Cold War », et cherchent à renouveler l'histoire des relations URSS/Est-Sud, dans différents domaines : économie, travail, médecine, art, culture mais aussi coopération éducative.

Genèse de la circulation des étudiants entre Afrique, URSS/Russie, pays de l'Est, Cuba, Chine

Les départs assez massifs d'étudiants africains vers l'URSs et les pays de l'Est ainsi que la création d'établissements destinés à les accueillir ne sont pas apparus subitement au début des années 1960 (Yengo 2011), et les contacts des Africains avec le marxisme-léninisme et le monde communiste sont anciens. Sans remonter à Abraham Hannibal, l'ancêtre de Pouchkine, venu en Russie vers 1704 et que l'on cite souvent comme le premier lien entre la Russie et l'Afrique, on voudrait au moins proposer quelques repères importants en partant de la Révolution de 1917. Celle-ci, aujourd'hui centenaire, ouvre une longue période où les nouvelles expériences révolutionnaires

12. Ce programme lancé par le Réseau interdisciplinaire Afrique Monde fin 2011 s'est développé dans le cadre de la Fondation Maison des sciences de l'Homme avec le soutien de l'Institut de recherche interdisciplinaire des enjeux sociaux (IRIS). M. Leclerc-Olive, P. Yengo et M. de Saint Martin en avaient la responsabilité.

13. Voir <http://socialismgoesglobal.exeter.ac.uk/>. 
jouent un rôle messianique dans le monde entier, y compris pour les étudiants; le premier pays socialiste est vu comme un défi lancé au modèle occidental.

Dans les années 1920 et 1930, plusieurs visiteurs africains et afroaméricains se rendent en URSS. «Entre Caraïbe, États-Unis, Europe, Afrique et URSS, il y eut très tôt, dès les toutes premières décennies du $\mathrm{XX}^{\mathrm{e}}$ siècle, une circulation d'idées et d'espérances qui prit une dimension qu'on dirait aujourd'hui globale ou encore cosmopolite» (Dozon 2015 : 14). C'est avec le Komintern que cette circulation est assurée. Des militants ou étudiants de différents pays sont reçus dans des écoles centralisées du Komintern créées à Moscou dans les années 1920, notamment à 1'Université communiste des travailleurs d'Orient (KUTV) pour les activistes des pays coloniaux.

Plusieurs anciens étudiants de la KUTV ont joué un rôle prépondérant dans la diffusion du marxisme en Afrique anglophone et lors des campagnes anticolonialistes des années 1930-1940 (Wilson 1974 : 123). Fondée par un décret du Comité central du 30 août 1921, celle-ci passe sous la direction du Komintern en juillet 1923, et forme de très nombreux militants et des révolutionnaires parmi lesquels une minorité du continent africain ; à partir de 1923, dix places sont réservées à l'accueil d'étudiants africains et afroaméricains qui doivent quitter leur pays de façon clandestine. Parmi les premiers élèves, d'ailleurs pas tous communistes, quelques-uns sont très engagés, comme Bankole Awoonor-Renner du Ghana ${ }^{14}$ ou Isaac WallaceJohnson du Sierra-Léone qui organise dans ce pays la West African Youth League, ainsi qu'Albert Nzula auquel Jean Copans consacre dans ce numéro une chronique bibliographique ${ }^{15}$. La plupart des étudiants de la KUTV sont peu instruits, parfois illettrés ; les femmes sont quasi absentes, ce qui traduit la place congrue qu'elles occupent dans la hiérarchie du Komintern, mais sans doute aussi parmi les militants anticolonialistes (Dullin \& Studer 2016). Avec l'École léniniste internationale, la KUTV a formé la plupart des dirigeants du Parti communiste sud-africain (Moses Kotane, John Marx, Albert Nzula, Edwin Mofutsanyana, secrétaire général du PC sud-africain dans les années 1932-1934) (Bertrand 2002: 186) ${ }^{16}$.

14. Bankole Awoonor-Renner entre à la KUTV en novembre 1925 et demeure à Moscou jusqu'en 1928. De son séjour à Moscou, il est resté un essai, Report on West Africa, qui expose un véritable projet de libération pour les colonies africaines ; ce texte inspire un document interne de trois pages détaillant un programme d'action en Afrique et décide le Komintern à faire de lui son envoyé en Afrique de l'Ouest. L'absence de toute trace de relations avec Moscou à partir de 1928 laisse cependant les chercheurs perplexes quant à son rôle d'agitateur au service de Moscou en Afrique de l'Ouest durant les trente années qui ont suivi (<http://maitron-en-ligne.univ-paris1.fr/spip.php?article159868>).

15. Jomo Kenyatta, futur président du Kenya, y effectua un séjour assez bref en 1932-1933.

16. La KUTV comptait environ 600 élèves en 1921, 1015 en 1924 (McClellaN $2007: 63,78)$. 
Albert T. Nzula, premier secrétaire noir du Parti communiste sud-africain, ancien instituteur et interprète dans un tribunal d'Afrique du Sud, arrive à la KUTV en 1931. Il y suit notamment les enseignements de deux professeurs renommés : Alexander Zusmanovich et I. I. Potekhin ${ }^{17}$, et est intégré aux travaux de la section pour les étudiants africains et afro-américains ainsi que du bureau africain qui recueillait des matériaux sur la situation politique et économique en Afrique; on y étudie les structures sociales en Afrique, les différentes formes de la lutte des classes, ainsi que les organisations politiques, notamment le Parti communiste d'Afrique du Sud.

Une collaboration pionnière et originale entre deux enseignants africanistes soviétiques et un étudiant africain ${ }^{18}$ — tous trois jeunes : Potekhin a 30 ans, Zusmanovich, 31 et Nzula, 28 - s'y développe et débouche sur un livre considéré comme l'un des premiers ouvrages russes publié sur l'Afrique à Moscou (à l'exception des travaux qui portent sur les origines du poète Pouchkine) sous le titre Le mouvement de la classe ouvrière et le travail forcé en Afrique nègre (Potekhin, Zusmanovich \& Jackson 1933) ; Jackson est le nom d'emprunt d'Albert T. Nzula ${ }^{19}$ qui meurt d'ailleurs l'année suivant la publication du livre à Moscou dans des conditions non encore élucidées.

Ce livre, dont Jean Copans relate l'histoire en même temps que celle de Nzula, n'a été traduit en anglais que quarante-cinq ans plus tard par Hugh Jenkins (1978) sous le titre Forced Labour in Colonial Africa ${ }^{20}$. Le livre est bien informé, en particulier sur l'Afrique du Sud, mais aussi sur le Ghana (Gold Coast), le Kenya, ou le Congo belge ; cependant, observe J. Copans, tout débat autour des démarches hétérodoxes ne se conformant pas au message de la III Internationale est exclu. L'existence de formations sociales originales en Afrique subsaharienne, notamment agricoles, y est suggérée, mais celle de classes sociales sur ce continent n'est pas véritablement discutée.

Dans les années 1930, les écoles du Komintern sont fermées l'une après l'autre. La politique d'accueil d'étudiants africains connaît un coup d'arrêt brutal avec la dissolution du Komintern en 1943 et la Seconde Guerre mondiale ${ }^{21}$.

L'événement qui signale le début de l'ère poststalinienne pour la politique culturelle de l'URSS et pour la multiplication des contacts et échanges avec le monde non communiste est sans aucun doute le $6^{\mathrm{e}}$ Festival mondial de

17. Potekhin est le premier africaniste soviétique à se rendre en 1957 en Afrique, au Ghana, et devient le premier directeur de l'Institut d'Afrique, fondé en 1959 (DAVIDSON \& IVANOVA 2003 : 37-39, 126).

18. Dans les pays occidentaux, c'est seulement quelques décennies plus tard que des enseignants publieront avec leurs étudiants africains (DAVIDSON \& FILATOVA 2007: 115).

19. Les militants communistes étaient alors forcés d'adopter de nombreux pseudonymes au gré de leurs diverses fonctions.

20. J. Copans a coédité un ouvrage avec R. Cohen et P. C. W. Gutkind (GutKInD, COHEN \& COPANS 1978).

21. En 1955, à la différence de la Chine, l'URSS n'a pas été invitée à la conférence de Bandung, événement fondateur du mouvement afro-asiatique. 
la jeunesse organisé à Moscou, du 28 juillet au 11 août 1957, avec 34000 participants étrangers venus de 131 pays, dont 600 Africains (Decraene 1959 : 414). Placé sous le signe de «la paix et l'amitié », ce $6^{\mathrm{e}}$ Festival est la plus grande manifestation organisée après-guerre dans le bloc communiste par la Fédération mondiale de la jeunesse démocratique ${ }^{22}$ et l'Union internationale des étudiants, avec un défilé au stade Lénine et une réception au Kremlin. Des organisations variées se côtoient dans un éclectisme qui tient non seulement aux relations privilégiées de l'URSS avec certains États, mais aussi à la neutralité politique affichée de l'événement. Le festival a de nombreuses répercussions sur la société moscovite en lui permettant d'entrevoir un mode de vie plus léger et décontracté, mais aussi sur les chercheurs africanistes soviétiques qui peuvent saisir une occasion de rencontres et d'échanges avec les étudiants ou militants africains, ce qui d'ailleurs vaut par la suite à Apollon Davidson l'interdiction de sortir d'URSS (Davidson \& Ivanova 2003 : 23). Il en a aussi sur les jeunes participants qui sont frappés par l'accueil triomphal qu'ils reçoivent, tels les deux délégués de la Fédération des étudiants d'Afrique noire en France (FEANF) qui rentrent de Moscou fortement impressionnés ${ }^{23}$. L'intérêt des autorités soviétiques pour la coopération avec l'Afrique est ensuite de plus en plus soutenu et se traduit par différentes mesures prises pour favoriser l'accueil des étudiants venus des pays africains et la formation d'une jeunesse africaine instruite.

Cependant, c'est la création en 1960 par Khrouchtchev de l'Université de l'amitié des peuples, rebaptisée Université Lumumba l'année suivante, qui constitue le point de départ d'une politique active de coopération éducative entre URSS et pays africains. Rapidement devenue le lieu d'une confrontation parfois forte entre gouvernement soviétique et gouvernements des pays africains, elle ne s'inscrit pas dans la continuité des universités du Komintern, et est plutôt conçue comme un symbole de l'internationalisme et de la solidarité de l'Union soviétique avec le tiers-monde. À l'heure de la « coexistence pacifique » érigée en principe de politique extérieure, l'objectif est d'établir un flux constant d'étudiants d'Afrique, d'Asie et d'Amérique latine vers l'Union soviétique, sans gêner la stabilisation des relations avec les nouveaux États, mais en espérant que ces jeunes, futures élites du Tiersmonde, trouvent dans leur pays leur propre «voie» du socialisme (Coumel 2001). Cependant, au lieu de devenir un lieu symbolique de discrimination positive, l'Université Lumumba est souvent fustigée par les gouvernements et les étudiants africains comme étant un établissement de discrimination ; dans les années 1980, elle est même devenue une université marginale (Katsakioris 2015 : 156-162).

22. La Fédération mondiale de la jeunesse démocratique (FMJD) est née dans l'après Seconde Guerre mondiale, lors d'une conférence internationale à Londres le 10 novembre 1945.

23. Deux délégués de la FEANF racontent dans L'étudiant d'Afrique noire l'accueil chaleureux du peuple soviétique, la grandeur du pays de Lénine (cités dans SADJI 2006 : 230-238). 
À l'image de l'Université Lumumba, la vie dans le «paradis soviétique » entraîne en effet de nombreuses désillusions. Portée par une propagande efficace au nom de l'amitié entre les peuples et évoquée par de nombreuses affiches, à l'instar de l'affiche de Vladimir Vasilevich Sachkov datant de 1964 où un étudiant soviétique et un étudiant africain se donnent la main devant l'Université Lomonossov (Fig. 1) ${ }^{24}$, elle n'échappe pas à la dure réalité du quotidien, émaillé de nombreux «incidents indésirables », comme les appellent les Soviétiques, en fait des agressions verbales et physiques contre les étudiants africains.

Au début des années 1960, trois étudiants africains sont morts dans des circonstances peu claires, ce qui provoque réactions et manifestation. Le 19 décembre 1963, une première et importante manifestation de protestation d'étudiants africains a lieu sur la Place Rouge après le décès d'un étudiant ghanéen, et a un grand retentissement parmi les étudiants mais aussi dans la presse africaine et occidentale. 500 à 700 étudiants, venant pour la plupart de l'Université Lumumba, y prennent part avec des slogans très forts: «Moscou, centre de la discrimination », «Arrêtez de tuer des Africains ! », ou «Moscou, une seconde Alabama», protestant en anglais, russe et français. Surtout, les étudiants africains mettent en œuvre des valeurs démocratiques, ce que les étudiants soviétiques n'osent pas faire (Hessler 2006). Inexistant pour les autorités soviétiques, y compris pour la police qui préfère parler de «bandes de hooligans » lorsque des étudiants africains sont attaqués, le racisme est présent dans les sociétés socialistes (Katsakioris 2015).

Jean-José Maboungou, dans sa chronique consacrée à deux ouvrages écrits par d'anciens étudiants africains ayant fait leurs études en URSS durant la période brejnévienne, permet d'appréhender ce racisme ordinaire et parfois violent de la société soviétique, à partir du roman de Zounga Bongolo, Un Africain dans un iceberg, consacré à l'histoire d'un amour impossible entre une jeune étudiante soviétique et un étudiant originaire du Congo-Brazzaville. Le second roman, Le gel, écrit dans un style très sobre,

24. Les affiches de propagande reprennent quelquefois des paroles de chansons populaires comme ici des vers de Vladimir Gusev sur une musique de Khrennikov: «Je n'oublierai jamais un ami, si je me suis lié d'amitié avec lui à Moscou. » Cette affiche de propagande historique, à la gloire de l'amitié entre étudiants africains et étudiants soviétiques, composée en 1964 par Sachkov Vladimir Vasilievitch, a été réimprimée à de nombreuses reprises et diffusée largement sur des sites internet, pour la plupart russes. Elle a été notamment présentée dans l'exposition « Red Africa » à la Calvert Foundation 22 qui s'est tenue à Londres du 4 février au 3 avril 2016. Né en 1928, l'artiste est diplômé de l'Institut des Beaux-Arts Sourikov à Moscou. Après avoir travaillé sur les affiches de films, il s'est orienté vers les affiches socio-politiques. Il a participé à des expositions au niveau national et international. Parmi ses affiches «La gloire héroïque des héros du Komsomol de la guerre froide ne sera jamais éteinte » (1967), "Parti — l'immortalité de notre projet» (1972), «Plan quinquennal de qualité - nos victoires sur le travail » (1975), « À l'appel du parti, à l'appel du cœur» (1977), «Le parti nous mène sur le chemin léniniste» (1978). 
FIG. 1. — « Je N'OUBLIERAI JAMAIS UN AMI SI JE ME SUIS LIÉ D’AMITIÉ AVEC LUI À MOSCOU », AFFICHE DE PROPAGANDE, 1964

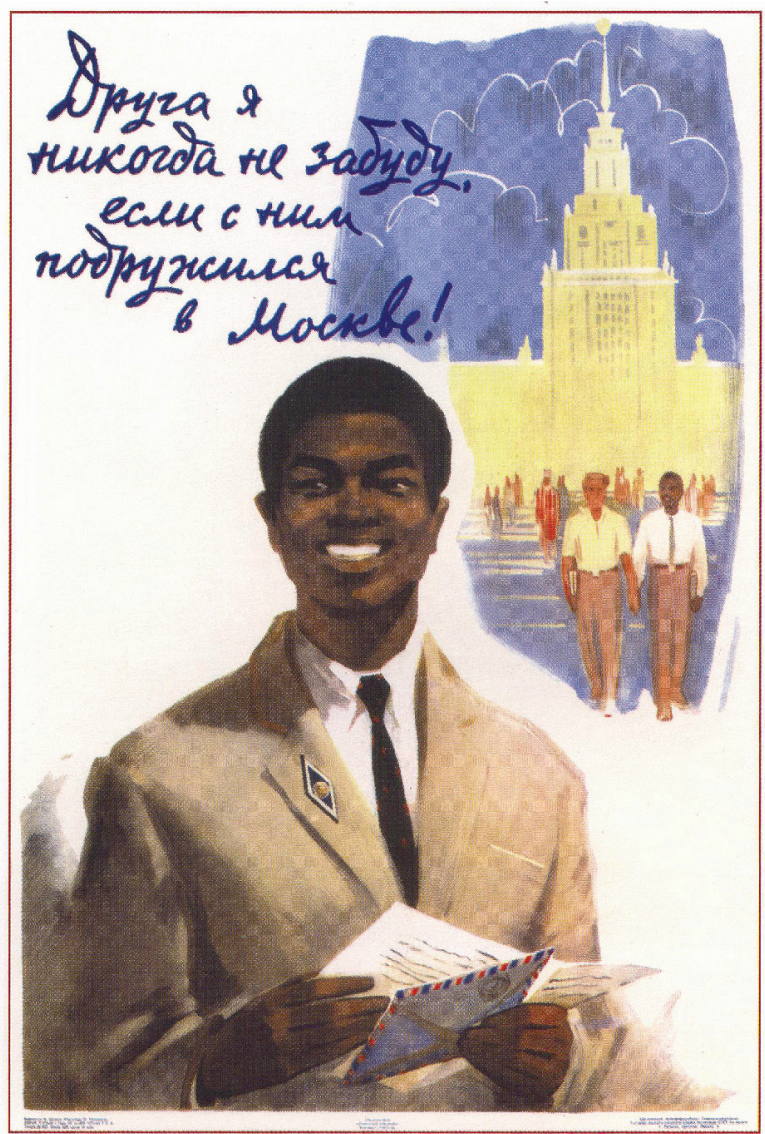

145. Сачков В.

Друга я никогда не забуду, если с ним подружился в Москве! 1964

est celui du grand écrivain Sonallah Ibrahim qui compose un exercice d'autofiction avec le journal de bord de Choukri, étudiant égyptien, observateur lucide des échecs et des tares du système soviétique à travers la vie quotidienne de ses camarades internationaux.

Construire un État indépendant. Retours et destins des élites formées à l'Est à l'aune des soubresauts des États africains

Il faut avoir à l'esprit que la notion d' « élite », en tant que catégorie sociale, n'était ni connue, ni reconnue comme telle en URSS, car elle contredisait l'idéologie de l'égalité sociale (Siim-Moskovitina \& Dobronravin 2015 : 
277). Son utilisation est aujourd'hui très large, mais sa définition est de fait sujette à variations et à désaccords (Heinich 2004). Elle est ici retenue dans une acception large pour désigner les milliers d'anciens étudiants et diplômés africains formés en URSS/Russie et dans les autres pays de l'ancien bloc socialiste qui ont exercé ou exercent aujourd'hui, à l'échelle nationale ou internationale, des fonctions très différentes : ingénieurs, agronomes, cadres moyens ou supérieurs de la fonction publique ou du secteur privé, médecins, pharmaciens, enseignants, cinéastes, artistes, hommes politiques, militaires. Loin d'une utilisation essentialiste de la notion, on s'intéresse à la grande diversité de ces élites qui, telles les élites éthiopiennes ou réunionnaises, ont beaucoup circulé entre l'Afrique, l'URss, l'Europe, l'Amérique du Nord et du Sud, et qui, parfois, ont aussi suivi une formation en Europe ou en Amérique.

L'une des singularités des formations des futures élites dans l'ex-bloc socialiste était qu'à l'issue des études, les diplômés étaient immédiatement renvoyés dans leur pays. Mais, en dépit de cette caractéristique des dispositifs de formation des étudiants étrangers, les devenirs de ces étudiants et stagiaires africains n'ont pas eu la linéarité prévue par cette description formelle. En outre, les retours donnaient lieu à des négociations inévitables, symboliques ou non, entre l'État et la nouvelle intelligentsia autour de la reconnaissance des diplômes et des régulations existant dans les pays de transit. Que ce soit à La Réunion, au Maroc, ou au Congo-Brazzaville, cette reconnaissance était souvent difficile à obtenir, et le parcours visant à avoir une équivalence avec le diplôme en vigueur dans le pays était semé d'embûches. Ainsi, à La Réunion, note Lucette Labache, il valait mieux faire allégeance au Parti communiste réunionnais si on voulait accéder aux postes les plus haut placés dans les mairies et administrations locales. Dans ces conditions, les retours dans le pays d'origine ont été souvent suivis de départs vers d'autres pays d'Afrique, Europe ou Amérique, suivis à leur tour de nouvelles tentatives de retour, sans compter celles pour rester sur place.

Les devenirs des anciens étudiants et diplômés restent de ce fait peu connus, et les sources sont fragmentaires et dispersées. Aucun suivi professionnel des cadres formés en URSS par exemple n'était assuré après leur retour dans leur pays, contrairement aux universités américaines ou britanniques (Siim-Moskovitina \& Dobronravin 2015 : 279). Dans les pays de départ, existent parfois des associations d'anciens étudiants passés par l'URSS/Russie qui tiennent des listes d'adhérents, par exemple au Sénégal ; en d'autres cas comme au Mali, il faut partir des archives du Bureau d'équivalences de diplômes ou parfois des services diplomatiques d'URSS/Russie, par exemple en Angola ou au Mozambique, qui ont entrepris dans les années 1980 des recherches assez officielles sur les anciens étudiants. Le fossé se comble petit à petit, et des travaux de plus en plus nombreux s'intéressent à cette histoire. C'est le cas des recherches de l'universitaire et diplomate kenyan Paul Kibiwott Kurgat dont la thèse intitulée Education as a Foreign Policy Tool: Kenyan Students Airlifts to USSR and Eastern Europe, 1854-1991 a 
servi de trame au livre qu'il a publié avec Alice Kurgat à Moscou (Kurgat \& Kurgat 2012).

Toutefois, le chantier de recherches ouvert depuis cinq ans est encore largement à explorer, d'autant plus que ce ne sont pas seulement des étudiants, des militants, des travailleurs, des militaires, des stagiaires ou de jeunes élèves africains qui étaient engagés dans ces mobilités. De nombreux coopérants ont été envoyés en Afrique par l'URSS, la RDA et d'autres pays socialistes (Goerg \& de Suremain 2014). Ainsi, Cuba a envoyé, à côté des quelques 2000 militaires, 50000 coopérants civils en Angola (Ribeiro 2014 ; Hatzky 2015). À l'inverse, des Africains, Mozambicains en l'occurrence, ont reçu une formation d'ouvriers spécialisés en République démocratique allemande qu'ils ont d'abord mise au profit de ce pays (Allina 2014). Il n'est pas certain que les expériences des uns et des autres se soient rencontrées; néanmoins ces différentes expériences, qui ont leur autonomie, participaient du même monde.

Méthodologiquement, la formation dans les pays du bloc socialiste soulève des questions de terminologie qui, à défaut d'être totalement résolues, permettent d'enrichir le débat. Il s'agit notamment de la question du statut des diplômés. Alors qu'en France, les étudiants africains étaient conscients d'être appelés à assumer la mission historique d'une nouvelle élite dirigeante (Guimont 1997), en était-il de même pour ceux qui étaient formés à l'Est ? Et si tant est qu'ils participaient des futures élites du pays à construire, étaient-ils idéologiquement marqués ? Y eut-il des élites « rouges », guidées par l'idéologie communiste et soucieuses de mettre en place une administration et une bureaucratie de type socialiste? Sans doute, mais fort peu. On croit souvent déceler dans la politique de l'Union soviétique et des pays du bloc socialiste une continuité entre, d'un côté, les écoles du Komintern, l'Université communiste des travailleurs de l'Orient, l'Université des travailleurs de Chine et, de l'autre, la formation des étudiants en URSS depuis 1960. Sous-entendu que, du Komintern à l'effondrement du mur de Berlin, la formation dans ces pays était mue par le désir de diffusion du communisme dans l'hémisphère sud, oubliant que, de l'enthousiasme qui a suivi la Révolution d'Octobre jusqu'à la pérestroïka, en passant par la période stalinienne et la déstalinisation puis la Guerre froide, le visage et les objectifs de la politique extérieure de l'Union soviétique avaient changé. Si l'URSS de Staline, obsédée par la sécurité, s'est intéressée à la formation des révolutionnaires pour exporter la Révolution à l'Orient, la période poststalinienne qui a élevé l'URSS au rang de grande puissance, l'assurant de la supériorité de son modèle, a contenu son influence dans les limites de la division du monde instituée à Yalta. Aux Indépendances, le modèle historique de la coopération éducative était moins la diffusion du communisme ou la formation de cadres « rouges » que l'éducation des étudiants des pays moins avancés à la modernité, dans le même esprit que dans les universités de l'Europe de l'Ouest. 
Le contexte historique était, comme le rappelle Constantin Katsiakoris ${ }^{25}$, « celui de la décolonisation et de la guerre froide, mais aussi celui du keynésianisme : de la foi dans le rôle absolument central de l'État dans le processus de développement économique et social, et de la conviction que l'État devait investir dans l'enseignement, car cet investissement dans les "ressources humaines" devait apporter beaucoup plus de bénéfices économiques que l'investissement direct dans la production. Le contexte intellectuel allait ainsi du keynésianisme occidental au socialisme soviétique et africain ».

On est ainsi loin du projet soviétique de faire de ces étudiants «non seulement des spécialistes hautement qualifiés au niveau de la science contemporaine, de la technique et de la culture, mais aussi des amis fidèles de l'Union soviétique, des propagandistes actifs des idées socialistes parmi leurs contemporains $»^{26}$. En effet, observe l'historien Sergey Mazov (2015 : 46), « visiblement, tous les étudiants étaient loin de brûler du désir de comprendre une doctrine qui déclarait que les réalités de leur pays étaient "des vestiges" ». Former des spécialistes hautement qualifiés était une tâche à la portée des Soviétiques, lesquels accordaient à leurs universités une confiance absolue. En revanche, faire des étudiants du Tiers-monde de véritables «amis de l'URSS », comme on aimait à le répéter, n'était ni donné, ni automatique, et s'avéra indéniablement le pari le plus complexe et le plus difficile à gagner (Katsakioris 2015). La réalité était que les étudiants avaient d'autres priorités et que même les nombreux communistes parmi eux résistaient à l'endoctrinement.

Un premier bilan de l'impact des mobilités vers les pays de l'ancien bloc socialiste sur le façonnement des États

Les devenirs et les parcours post-études des anciens étudiants ayant suivi des études supérieures dans un des pays du bloc socialiste sont très variés et contrastés; inutile de chercher à dresser un portrait ou un profil type. En effet, il existe une grande diversité de situations, depuis quelques grandes figures du monde politique, de la diplomatie ou du cinéma, jusqu'à ceux et celles qui ont dû exercer des emplois précaires, en passant par les très nombreux ingénieurs et cadres techniques, de sorte qu'il est difficile de présenter un bilan des contributions des mobilités/migrations des étudiants dans les pays de l'ancien bloc socialiste, notamment au façonnement des États. Les retours dans le pays d'origine après l'obtention du diplôme s'avèrent

25. Intervention «La formation des cadres/quadros africains en URSS : le cas des étudiants des colonies portugaises, 1960-1974 » au séminaire ÉLITAF, 3 février 2015, non publié.

26. RGANI. F. 4. Inv. 16. D. 902. F. 34. Ordre du ministre de l'Enseignement supérieur et secondaire spécialisé de l'URSS, $\mathrm{n}^{\circ} 86$, «Le travail d'éducation parmi les étudiants et thésards venus en URSS des pays capitalistes peu développés sur le plan économique », 30 juin 1960, cité par MAzov (2015: 43). 
bien compliqués pour beaucoup de diplômés dès le début des années 1980 . Plusieurs États d'Afrique, frappés par les plans d'ajustement structurel ne peuvent plus garantir l'embauche des jeunes professionnels. Alors, certains se dirigent vers l'Europe de l'Ouest ou le « nouveau monde », souvent après avoir tenté de trouver un poste en Russie ou en Afrique. Les itinéraires suivent des logiques de plus en plus « globales » de valorisation de diplômes et de compétences (Dimitrova 2015 : 184).

Toujours est-il que, de retour au pays, le nouveau diplômé est parfois tiraillé entre deux postures. Il s'agit pour lui de s'engager soit comme acteur de terrain dans la tâche que commande la formation reçue (médecin, pharmacien, vétérinaire, ingénieur, agronome, technicien, etc.), soit dans la consolidation de l'appareil d'État sur ses deux versants, politique et administratif. Même si la promotion dans l'appareil d'État offre plus de visibilité, le succès de la formation dans les pays de l'Est repose en fait essentiellement sur le grand nombre de nouvelles élites, anonymes, engagées sur le terrain ; qu'elles soient des cadres supérieurs, intermédiaires ou techniques. Cependant, les deux postures ne se repoussent pas forcément ; elles peuvent même être complémentaires, et les nouveaux diplômés sont souvent sollicités pour occuper aussi des postes administratifs ou politiques, ce qui peut obérer leurs compétences. En effet, la première alternative reste subordonnée à la seconde en ce sens que le champ d'intervention d'un technicien sur le terrain ou l'affectation d'un médecin ou d'un vétérinaire dans une région est indissociable de la voie politique dans laquelle le pays s'engage. Pour les régimes se réclamant du socialisme, rompre avec les rapports de domination politique et économique issus du colonialisme ou des structures traditionnelles locales était un enjeu de taille dont le volontarisme pouvait conduire à des actions violentes suscitant de fortes réactions de la part des dépossédés.

Dans les premières années de la souveraineté nationale, l'indépendance économique du pays reposait sur le secteur d'État national dont le rôle était alors contradictoire. D'un côté, celui-ci restait soumis à un appareil de production qui s'était maintenu dans les mêmes lignes qu'avant l'indépendance. Il se mettait alors à la remorque des grands groupes privés dont il garantissait l'extension dans les secteurs agricoles, miniers ou d'industrialisation de pointe. De l'autre, il tentait de desserrer l'étau de la propension trop grande de ces entreprises privées à l'accaparement des ressources en y prenant des participations. Si dans ce second cas, la participation de l'État était une caution pour les entreprises à la fois contre les revendications des salariés et pour la poursuite d'une politique entrepreneuriale dans les termes des monopoles industriels, elle pesait moins sur le recrutement des cadres. Dans le secteur industriel qui dépendait presque exclusivement de l'extérieur, c'était le détenteur des capitaux privés qui dictait la politique d'embauche. Les compétences recherchées étaient celles que validait la formation dispensée dans les lieux d'où provenaient les capitaux, en l'occurrence les pays occidentaux. C'est pour cette raison que seul le secteur d'État garantissait le 
recrutement des cadres formés à son initiative dans les pays du bloc socialiste non sans avoir testé leurs compétences ${ }^{27}$.

Reste que pour l'État, seul son appareil était en mesure d'absorber les cadres dont il avait incité et supervisé la formation dans les États socialistes. La suppression du multipartisme et la création du parti unique ont fait du nouveau parti «national » l'un des points de chute des anciens étudiants en URSS et dans les pays socialistes auxquels étaient attribuées les tâches de sa politique d'information et de propagande. De manière générale, leur premier employeur était l'appareil politique. Ces élites en provenance d'URSS et des pays du bloc socialiste étaient en effet intégrées dans le parti ou le gouvernement ou affectées aux fonctions régaliennes du pays (défense et sécurité).

En l'absence de recherches proposant un panorama historique du devenir des militaires africains formés en URSS, l'article de Natalia Krylova nous permet d'accéder à l'un des principaux lieux de leur formation, le centre-165 Perevalnoe en Crimée. Initialement prévu pour les combattants des mouvements de libération, celui-ci s'est transformé au fil du temps en une école de formation pour les cadres militaires supérieurs à mesure que les États accédaient à leur indépendance. La formation de militaires dans les nombreux centres et académies constituait l'un des principaux volets de la coopération militaire et militaro-technique de l'Union soviétique qui a permis aux jeunes États de se doter d'armées nationales comme attribut de leur souveraineté sans rompre pour certains leurs relations avec les anciennes puissances coloniales.

En ce qui concerne les élites politiques les plus connues et reconnues, quelques noms d'anciens étudiants sont souvent cités dans les travaux des chercheurs : José Eduardo dos Santos, président de l'Angola depuis 1979, président du MPLA, a étudié en URSS de 1963 à 1969 ; il était alors le principal dirigeant des étudiants angolais en URSS et a obtenu à Bakou un diplôme d'ingénieur du pétrole et des télécommunications. Fikre-Selassié Wogderess, Premier ministre éthiopien de 1985 à 1987, à la fin du Derg, a étudié à l'Institut de sciences sociales à Moscou en 1975 ; Alemu Abebe, ministre de l'Agriculture en Éthiopie a fait des études de médecine vétérinaire à Moscou. Au Mali, plusieurs présidents ont été formés en URSS ou en Europe de l'Est, parmi lesquels Alpha Oumar Konaré (1971-1975 : Institut d'Histoire, Université de Varsovie), Amadou Toumani Touré (1974-1975 : École supérieure des troupes aéroportées à Riazan en URSS), Dioncounda Traoré (1962-1963 : Faculté de langue russe à Moscou ; 1963-1965 : Faculté de mécanique et mathématiques de l'Université d'État de Moscou). Pendant ses études à Moscou, ce dernier a aussi été secrétaire à la presse de la Société des élèves et étudiants maliens en URSS, et ensuite secrétaire politique de l'Association

27. C'est dans ce sens qu'il faut comprendre les stages de mise à niveau dont témoignent nombre de diplômés et que l'on justifie souvent par une homogénéisation des nomenclatures entre la langue d'étude et la langue administrative (portugais, français, anglais) du pays (TCHICAYA-OBOA \& YENGO 2015). 
des élèves et étudiants maliens en URSS (Siim-Moskovitina \& Dobronravin 1995 : 283). Les diplomates sont aussi nombreux; ainsi, le réseau des missions diplomatiques béninoises à l'étranger compte aujourd'hui 14 ambassadeurs anciens étudiants en URSS et dans les pays de l'Est. Le plus emblématique d'entre eux est Anicet Gabriel Kotchofa, diplômé de l'Institut Goubkine du pétrole et du gaz de Moscou, qui a effectué toute sa carrière professionnelle dans les universités russes avant de devenir ambassadeur du Bénin à Moscou. Cependant, au sommet de l'État, ce sont souvent les diplômés des pays occidentaux qui ont occupé les positions les plus importantes.

Dans le domaine culturel, la formation aux différents métiers du cinéma (opérateurs, scénaristes, réalisateurs, critiques de cinéma, éclairagistes, etc.), que ce soit sur le continent africain, en Union soviétique ou dans d'autres pays de l'Est, retient fortement l'attention; le cinéma jouait en effet un rôle essentiel dans la conquête des cœurs et des esprits. Parmi les cinéastes, le Sénégalais Sembene Ousmane, le Malien Souleymane Cissé, l'Éthiopien Micha Papatakis, l'Algérien Azzedine Meddour ou encore le Mauritanien Abderrahmane Sissako, pour n'en citer que quelques-uns, ont fait leurs classes en Union soviétique, pour la plupart à l'Institut du cinéma de Moscou (VGIK). La formation soviétique a joué et joue encore un rôle majeur dans la manière de représenter leur société ; revenus dans leurs pays respectifs, les cinéastes africains formés en URSS ont souvent voulu montrer la vie quotidienne de leurs concitoyens. Même peu nombreux, ces cinéastes ont marqué leur cinématographie nationale, voire mondiale; plusieurs d'entre eux ont constitué les bases institutionnelles d'un cinéma national encore présent aujourd'hui dans leurs pays (Chomentowski 2016).

Une page moins connue de la coopération culturelle entre les pays du bloc socialiste et l'Afrique est celle qui concerne les arts plastiques, la sculpture et la peinture notamment. Nora Greani nous en donne un aperçu avec le Congo-Brazzaville. Dans son article, elle aborde la question de l'art mis au service du Parti congolais du travail et des moyens mis en œuvre pour construire un art socialiste révolutionnaire : formation des artistes en URSS et en Chine, mise en place de structures institutionnelles (notamment l'Union nationale des écrivains et des artistes congolais, sur le double modèle de l'UNEAC cubaine, et de l'Union des artistes soviétiques). On imagine aisément que le Congo-Brazzaville ne fut pas le seul pays africain à être tenté par le «réalisme socialiste» mis au service des masses laborieuses. Mais on imagine aussi aisément qu'un continent profondément marqué par une représentation artistique au fort accent symbolique ne se soit pas facilement accommodé de ce rétrécissement de l'imaginaire.

Si quelques-uns des anciens étudiants dans les pays du bloc socialiste ont obtenu une très grande reconnaissance nationale, voire internationale, dans le monde politique ou dans la culture, ce sont surtout des milliers d'ingénieurs, agronomes, médecins, pharmaciens, cadres de l'administration et du secteur privé, techniciens, enseignants d'université ou du secondaire, parmi lesquels les femmes étaient minoritaires, qui ont contribué à constituer 
la bureaucratie administrative et l'encadrement technique dans les entreprises des pays d'où ils étaient partis ou ont permis d'ouvrir des cabinets privés de médecin ou des officines de pharmacien en des lieux qui en étaient dépourvus. Les Africains retournés dans leur pays travaillent aujourd'hui de plus en plus dans des compagnies russes ou comme intermédiaires entre les hommes d'affaires russophones de l'ancienne URSS et les milieux commerciaux et sociaux et l'appareil d'État de leur pays, par exemple dans les grandes compagnies d'extraction de ressources en énergie telles que RusAl en Guinée.

Pour être les deux premiers pays africains à s'être revendiqués ouvertement du marxisme-léninisme, le Congo-Brazzaville et le Bénin occupent une place particulière en ce qui concerne le recours aux élites formées dans les pays de l'ex-bloc soviétique. Avec un premier convoi d'étudiants parti pour l'URSS en 1965, le Congo compte dans son personnel politique de nombreux anciens étudiants de l'Europe de l'Est. Leur présence était déjà signalée dans les rouages de l'État avant le discours de la Baule de François Mitterrand et la vague des conférences ayant mis fin au parti unique. Mais c'est au lendemain de la guerre civile - au cours de laquelle beaucoup d'entre eux ont joué un grand rôle aux côtés de Sassou-Nguesso dont ils ont assuré la victoire et le retour au pouvoir - que leur prégnance est devenue manifeste (Yengo 2006). Outre les cadres administratifs et techniques des entreprises d'État, l'on compte parmi les figures les plus marquantes du pays quelques anciens diplômés des pays du bloc socialiste qui se sont juchés au sommet de l'État. Isidore Mvouba, ancien étudiant de l'Institut des chemins de fer de Moscou de 1971 à 1976, ingénieur, a été ministre des Transports de 1999 à 2002, puis ministre d'État, ministre des Transports et des Privatisations, chargé de la coordination de l'action gouvernementale de 2002 à 2005, et enfin Premier ministre de 2005 à 2016. Henri Djombo, diplômé de l'Institut des eaux et forêts de Leningrad, a été reconduit au poste de ministre de l'Économie forestière de 1997 à 2016 avant d'être nommé ministre d'État chargé de l'Agriculture, de l'Élevage et de la Pêche. François Ibovi, qui a été ministre de la Communication, chargé des relations avec le Parlement et porte-parole du gouvernement de 1997 à 2002, puis ministre de l'Administration territoriale et de la Décentralisation (2002-2007) et enfin ministre de la Santé et de la Population (2012-2016) est un journaliste formé à l'Université Lomonossov de Moscou de 1973 à 1979. Le philosophe Charles Zacharie Bowao, formé à Moscou, a occupé successivement les postes de ministre chargé de la Coopération, de l'Action humanitaire et de la Solidarité (2007-2009) puis de ministre chargé de la Défense (2009-2012). La liste n'est pas exhaustive et on peut y ajouter les noms de Mamadou Camara Dekamo, ambassadeur du Congo en Italie, ou de Paul Gatsé-Obala, ancien ambassadeur au Gabon.

Le Bénin est le deuxième pays subsaharien à avoir connu un régime marxiste-léniniste de 1974 à 1991, et a pu recruter un peu plus de 2500 cadres $^{28}$

28. Chiffre avancé par Anicet Gabriel Kotchofa (voir supra), <http://www.benin. mid.ru/fr/gabriel.html>. 
formés en URSS/Russie, en sciences médicales, art, sciences de l'ingénieur, journalisme et autres disciplines. À leur retour au pays, les élites béninoises formées dans les pays de l'ancien bloc socialiste ont pour la majeure partie bénéficié de l'État providence et notamment d'une intégration directe dans la fonction publique, du moins jusqu'au gel des recrutements directs dans la fonction publique en 1985 lors des premières pressions des organisations financières internationales : FMI et Banque mondiale. Les premiers diplômés ont intégré l'administration béninoise et les grandes entreprises publiques : nombre d'entre eux ont occupé des positions privilégiées dans les ministères techniques (énergie, eau, communications, etc.), ou encore dans les grandes entreprises d'État d'hydraulique et d'énergie (Société béninoise d'énergie électrique, Société nationale des eaux du Bénin, toutes deux réunies jusque récemment au sein d'une seule et même entreprise, l'ex Société béninoise d'eau et d'électricité, etc.) ou dans les sociétés d'information et de communication (Office de radiodiffusion et de télévision du Bénin, Office national d'imprimerie et de presse). Plusieurs sont entrés dans l'enseignement, notamment dans les écoles supérieures et universités privées en plein développement, par exemple à l'École supérieure de génie civil Verechaguine A. K, mais aussi à l'École polytechnique de l'Université d'Abomey-Calavi, la plus grande université publique. D'autres ont ouvert des cabinets privés de médecine et d'architecture. Si la formation d'étudiants dans les instituts techniques et les universités des pays de l'Est a été un facteur majeur de la production des cadres administratifs dans les années 1980 et 1990, ceux-ci sont restés paradoxalement peu visibles dans les arènes politiques (Eyebiyi 2012).

Ces contingents importants qui sont parvenus à occuper des postes techniques et parfois administratifs ne sauraient faire oublier les déclassés dont le diplôme n'a été ni reconnu, ni évalué à sa juste valeur. Ceux-ci ont dû survivre ou survivent encore avec des emplois modestes de cadres moyens, lorsque ce n'est pas avec des petits boulots ou le chômage. En Éthiopie, par exemple, d'anciens officiers du régime du Derg sont occupés à lever des barrières à l'entrée de l'aéroport ou à des emplois de gardiens, sans parler de ceux qui ont été éliminés de façon tragique dans les pays frappés par des révolutions ou des changements brusques de régime politique.

Alors que les Éthiopiens ayant fait des études en URSS occupaient une part importante des hautes fonctions au ministère des Affaires étrangères et dans les ministères économiques, les commissions et les entreprises d'État, durant le régime du Derg, le changement intervenu suite au renversement du gouvernement de Mengistu Haile-Mariam et à la prise de pouvoir par le Front démocratique révolutionnaire du peuple éthiopien en 1991 modifia profondément cet état de fait; les renvois et parfois les éliminations des anciens hauts fonctionnaires furent nombreux. Dans ce cas, il semble bien que les élites «retournantes » de l'étranger, et notamment celles passées par l'URSS et les pays de l'Est n'aient pas réussi à s'inscrire de manière importante et durable dans le paysage politique éthiopien où la domination 
des élites «locales » tirant leur légitimité principalement de leur ancrage politique, religieux, culturel et économique dans le pays est encore forte selon l'hypothèse proposée par l'article de Tassé Abye.

$\mathrm{Au}$ Maroc, pays éloigné idéologiquement du communisme, les anciens diplômés des établissements d'enseignement supérieur soviétiques/russes sont très nombreux aujourd'hui à exercer des professions spécialisées (pharmaciens, ingénieurs), mais ils n'ont pas pu occuper des positions très élevées dans les administrations publiques et le secteur économique du pays, comme cela a été le cas dans plusieurs pays africains au sud du Sahara (Mellakh 2016).

Le cas des Touaregs, boursiers de l'État du Mali, diplômés d'URSS ou d'un autre pays du bloc de l'Est, est très éclairant. S'ils n'ont pas connu de ségrégation à proprement parler et si plusieurs d'entre eux ont réussi à intégrer la fonction publique malienne ou le secteur privé, les postes politiques de haut niveau ou les postes dans la haute administration confiés aux Touaregs ont été surtout occupés par des anciens des écoles « occidentales».

À La Réunion, département d'outre-mer depuis 1946, une filière politique de migration mise en place dès les années 1960 par le Parti communiste réunionnais, dont Paul Vergès était le secrétaire général, envoya des jeunes Réunionnais faire des études supérieures de médecine, agronomie, physique, mathématiques, lettres et langues étrangères dans les pays de l'ancien bloc de l'Est afin de former des cadres et des élites pour soutenir le futur État autonome. Si les étudiants réunionnais, souvent sympathisants du PCR, sont partis par cette filière avec beaucoup d'enthousiasme et un projet politique, ils ont souvent connu des désillusions, d'abord en URSS, plus encore au retour sur l'île où leurs diplômes n'étaient pas toujours reconnus et où souvent les postes qui leur étaient proposés ne correspondaient pas à leur qualification ; d'ailleurs plusieurs sont partis vers l'Afrique continentale. Lorsque les conseils général et régional ont été dirigés par la gauche, plusieurs anciens de l'Est ont occupé des postes à responsabilité comme directeurs des services ou chargés de mission au sein de ces collectivités, mais aucun n'a occupé de poste de pouvoir dans la haute administration ou dans les grandes entreprises.

Ainsi, dans nombre de cas dans les pays nouvellement indépendants ou à la recherche de l'autonomie, est apparue une distinction nette entre, d'une part, les professions administratives, politiques et financières - dont les formations ont, durant la Guerre froide, continué d'être assurées en grand nombre dans les anciens États colonisateurs - et les professions d'ingénieur, technicien, ou de médecin, pharmacien qui étaient demandées aux pays de l'Est. Au sommet de l'État, c'était la formation occidentale, toutes professions confondues, qui primait, et ce même pour les corps d'armée et les services de sécurité que certains pays de l'Est ont pourtant contribué à professionnaliser. Au point que la promotion interne invitait nombre de cadres formés dans l'ex-bloc soviétique à se recycler, à un moment ou un 
autre, en France ou en Royaume Uni. Car, à compétences égales ou à statut équivalent, le privilège de la légitimité revenait à ceux qui avaient étudié dans le pays colonisateur. Dans la hiérarchie des diplômes, le parchemin délivré dans les pays occidentaux et, particulièrement, par l'ancienne puissance coloniale, avait selon toute vraisemblance une plus grande valeur. Fafali Koudawo, l'un des premiers à s'intéresser aux cadres « révolutionnaires » formés en URSS, faisait remarquer il y a déjà vingt-cinq ans à propos du Togo, que si les anciens diplômés d'URSS étaient souvent dans les cercles du pouvoir, ils étaient parfois victimes de railleries de la part de leurs compatriotes qui les affublaient de surnoms tels que « Russotiques » ou « Moscoutiques » (Koudawo 1992).

L'idéologie du progrès qui a accompagné les Indépendances et a permis d'ouvrir les pays africains tantôt sur le modèle européen, qu'il soit de l'Ouest ou de l'Est, tantôt sur le modèle asiatique, marquant ainsi des préoccupations à caractère pragmatique, est avant tout fondée sur la recherche d'une indépendance réelle. L'indépendance est la réponse idéologique africaine à l'interventionnisme non moins idéologique des deux blocs qu'Odd A. Westad (2005) a découvert dans la prétention des États-Unis comme de l'URSS à justifier leur action dans le Tiers-monde par le concept de modernité hérité des Lumières.

C'est peut-être cette quête d'indépendance qui a guidé l'action des dirigeants africains au lendemain des Indépendances. La voie prise par les uns ou par les autres importe peu, et les régimes africains qui se sont réclamés du socialisme sont aussi divers que les modèles dont ils pouvaient se revendiquer. Des modèles prétendant au «socialisme réel » existaient, qu'il s'agisse de l'URSS, des démocraties populaires, de la Chine ou de Cuba, offrant aussi une panoplie réelle ou fantasmée, prête à servir. Au-delà de ces exemples étrangers que l'on a parfois tenté d'imiter ou d'adapter, il y a eu l'invention d'un socialisme qui se voulait adapté à l'Afrique ${ }^{29}$.

La réflexion sur les spécificités du continent en puisant dans son passé les racines d'un projet de développement de type socialiste a inspiré beaucoup de dirigeants, de Nyerere Julius à Léopold Sédar Senghor. Ceux qui ont cherché leur inspiration dans les outils du marxisme-léninisme ne sont pas légion. Parmi eux, on compte Marien Ngouabi pour le Congo-Brazzaville, Matthieu Kérékou pour le Bénin, Mengistu Hailé Mariam pour l'Éthiopie. Autant de dirigeants de régimes militaires qui ne pouvaient survivre à l'effondrement du modèle soviétique et à l'imposition de la logique libérale des programmes d'ajustement structurel (Chossudovsky 1997).

29. Voir le colloque sur «Les socialismes africains/Socialismes en Afrique » organisé par Françoise Blum à Paris du 7 au 9 avril 2016. Les travaux de ce colloque doivent donner lieu à une publication (voir l'appel à communications sur <http:// chs.univ-paris1.fr/AppelacomSocialismeafricain2.pdf $>$, et le programme notamment sur <https://www.ehess.fr/fr/colloque/socialismes-africains-socialismes-enafrique $>$ ). 
En définitive, si quelques anciens étudiants dans les pays socialistes ont fini par compter parmi la minorité dirigeante des États, dans quelle mesure ont-ils vraiment contribué à leur structuration ? Cette interrogation surgit en effet au creux de la configuration institutionnelle des États post-coloniaux qui invite à saisir la nature de l'objet étatique à partir de ses assignations politiques dans la division du monde et en fonction de logiques de prise de décision politique dans leur globalité. En ce sens, pour comprendre qui gouverne dans une configuration post-coloniale, il ne faut pas se baser uniquement sur les figures instituées du pouvoir d'État et sur les élites qui, au cœur de l'État, mettent en œuvre les programmes de politiques publiques, mais il faut appréhender la relation État/élites dans l'ensemble du rapport de domination à l'échelle mondiale, des modes de reproduction et d'autolégitimation du pouvoir d'État. Il ne faut pas ignorer non plus que le pouvoir décisionnel (même au cœur de l'État) est partagé entre les élites économiques souvent en position d'extraversion et les grands corps militaires et administratifs dont la légitimité est assurée par les anciennes puissances coloniales. Dans de telles configurations, les élites du pouvoir, même quand elles investissent dans l'État, ne possèdent pas la capacité d'en modifier la nature, et les proclamations les plus « anti-impérialistes » auxquelles nous ont habitués les régimes « marxistes-léninistes » n'y peuvent rien. D’une part, parce qu'il n'y a aucune uniformité des élites, puisque le système de recrutement au sommet dépend de la nature du régime. D'autre part, parce que dans ces pays où les élites sont aussi soumises à « la tyrannie des diplômes » (Bauer \& Bertin-Mourot 1995), le lieu d'émission de ces diplômes compte pour beaucoup dans leur adoubement. Étant donné que la plupart des régimes africains, dans la période qui nous intéresse (des Indépendances à la chute du mur de Berlin) étaient autocratiques, les solidarités élitaires y étaient structurées à partir du parti unique et de l'allégeance à la figure du chef de l'État. Or, dans la mesure où le parti unique participait de la promotion politique, la confrontation entre les professionnels de la représentation politique et les élites techniques en quête d'efficacité était inexorable. De ce point de vue, et au-delà des «success stories » mentionnées, les élites formées dans les pays du bloc socialiste apparaissent plutôt comme agissantes en-deçà de l'élite dirigeante du pouvoir d'État, œuvrant notamment dans l'anonymat des administrations, des travaux publics, des hôpitaux ou en faisant le choix des politiques publiques. Mais comme le montrent les exemples de l'Éthiopie et de l'Angola, c'est au moment où disparaît la bipolarité Est-Ouest que le paradoxe de la situation des élites devient manifeste. Fragilisées par le tournant historique, elles sont devenues les actrices du dessaisissement de leur propre histoire qu'elles tentent désormais de dissimuler.

Institut des mondes africains (IMAF), EHESS, Paris;

Institut de recherche interdisciplinaire sur les enjeux sociaux (IRIS), EHESS, Paris. 


\section{BIBLIOGRAPHIE}

Allina, E.

2014 «Labor on the Frontline: Mozambican Workers in East Germany, 1979$1990 »$, non publié, téléchargeable avec autorisation de l'auteur sur <https: //www.academia.edu/9945587/>.

BAuer, M. \& Bertin-Mourot, B.

1995 « La tyrannie du diplôme initial et la circulation des élites : la stabilité du modèle français », in E.-N. Suleiman \& H. MEndRas (dir.), Le recrutement des élites en Europe, Paris, La Découverte : 48-63.

BAYART, J.-F.

1989 L'État en Afrique. La politique du ventre, Paris, Fayard («L'espace du politique $»)$.

BERTRAND, F.

2002 L'anthropologie soviétique des années 20-30. Configuration d'une rupture, Pessac, Presses universitaires de Bordeaux.

BOLTANSKI, L.

2009 De la critique. Précis de sociologie de l'émancipation, Paris, Gallimard.

Boltovskaja, S.

2014 Bildungsmigranten aus dem subsaharischen Afrika in Moskau und St. Petersburg: Selbst- und Fremdbilder (Migrants d'Afrique subsaharienne en quête de formation à Moscou et Saint-Pétersbourg : Images de soi et d'autrui), Herbolzheim, Centaurus Verlag \& Media UG.

Boulland, P. \& Gouarne, I.

2015 «Les mondes mêlés du communisme : une autre approche transnationale?», Critique internationale, 66 (1) : 9-18.

BREDELOUP, S.

2014 «Pluralité des parcours des étudiants ouest-africains en Chine», Cahiers de la recherche sur l'éducation et les savoirs, 13: 139-165.

Chomentowski, G.

2016 «L'expérience soviétique des cinémas africains au lendemain des indépendances », Le temps des médias, 26 (1) : 111-125.

Chossudovsky, M.

1997 The Globalization of Poverty: Impacts of IMF and World Bank Reforms, Penang, Third World Network; London, Zed Books.

Coumel, L.

2001 «Moscou, 1960: la fondation de l'Université de l'Amitié des Peuples », Bulletin de l'Institut Pierre Renouvin, 12, <https://www.univ-paris1.fr/autresstructures-de-recherche/ipr/les-revues/bulletin/tous-les-bulletins/bulletin-n12-grandes-villes-et-relations-internationales/laurent-courmel-moscou-1960la-fondation-de-luniversite-de-lamitie-des-peuples/>. 
Davidson, A. \& Filatova, I.

2007 «African History: A View behind the Kremlin Wall», in M. Matusevitch (ed.), Africa in Russia. Russia in Africa. Three Centuries of Encounters, Trenton N.J.-Amsara, Africa World Press : 111-131.

DAvidson, A. \& Ivanova, L.

2003 Moskovskaya Afrika. Prirodnoe kulturnoe nasledie Moskvui (L’Afrique Moscovite. L'héritage culturel de Moscou), Moskva, Izdatelstvo Teatrlanogo Instituta Imeni Borisa Shukina.

DeCRAene, P.

1959 «Pan-africanisme et grandes puissances », Politique étrangère, 24 (4) : 408421.

Dimitrova, S.

2015 «Étudiant international en URSS, Africain en Russie, citoyen global au Québec. Expériences de transition », in M. DE Saint Martin, G. Scarfò Ghellab \& K. Mellakh (dir.), Étudier à l'Est. Expériences de diplômés africains, Paris, Karthala-FMSH : 173-185.

Dozon, J.-P.

2015 «Préface», in M. de Saint Martin, G. Scarfò Ghellab \& K. Mellakh (dir.), op. cit. : 11-15.

Dullin, S. \& Studer, B.

2016 «Communisme transnational. L'équation retrouvée de l'internationalisme au premier $\mathrm{XX}^{\mathrm{e}}$ siècle », Monde(s). Histoire, espaces, relations, 10, novembre : 9-32.

EYeBiyi, E. P.

2012 «De la pertinence du Bénin comme terrain d'études», in ÉLITAF, Programme de recherche Étudiants et élites africaines formés dans les pays de l'ex-bloc soviétique: histoires, biographies, expériences, Document de travail interne: 30-36, Paris (littérature grise, non publié).

Eyebiyi, E. P. \& MAZZella, S.

2014 «Introduction : Observer les mobilités étudiantes Sud-Sud dans l'internationalisation de l'enseignement supérieur », Cahiers de la recherche sur l'éducation et les savoirs, $13: 7-24$.

Gheorghiu, M. D. \& Netedu, A.

2015 «Étudiants d'Afrique en Roumanie et en RDA. Les cadres sociaux et politiques de leurs expériences », in M. de Saint Martin, G. Scarfò Ghellab \& K. Mellakh (dir.), op. cit. : 95-109.

GinZBuRG, C.

2009 «Peur, révérence, terreur. Lire Hobbes aujourd'hui », MethIS, 2 : 23-47, $<$ http://popups.ulg.ac.be/2030-1456/index.php?id=285\&file=1\&pid=277>. 
Goerg, O. \& de Suremain, M.-A. (Dir.)

2014 «Coopérants et coopération en Afrique : circulations d'acteurs et recompositions culturelles (des années 1950 à nos jours) », numéro spécial, OutreMers. Revue d'histoire, 102 (384-385).

Gómez Martin, C.

2016 «La génération sahraouie de la guerre : des études à Cuba à la migration économique en Espagne ", Revue européenne des migrations internationales, $32(2)$ : 77-94.

GóNZALEZ LóPEZ, D.

2008 «Impactos de Africa en Cuba », Temas, 56 : 29-37.

Guimont, F.

1997 Les étudiants africains en France (1950-1965), Paris, L'Harmattan.

Gutkind, P. C. W., Cohen, R. \& Copans, J. (eds.)

1978 African Labor History, Beverley Hills-London, Sage Publications.

Hatzky, C.

2015 Cubans in Angola: South-South Cooperation and Transfer of Knowledge, 1976-1991, Madison, The University of Wisconsin Press.

HeINICH, N.

2004 «Retour sur la notion d'élite», Cahiers internationaux de sociologie, 117 (2): 313-326.

HESSLER, J.

2006 «Death of an African Student in Moscow. Race, Politics, and the Cold War», Cahiers du monde russe, 47 (1) : 33-63.

KARADY, V.

2002 «La migration internationale d'étudiants en Europe, 1890-1940», Actes de la recherche en sciences sociales, 145 (5) : 47-60.

KATSAKIORIS, C.

2015 Leçons soviétiques. La formation des étudiants africains et arabes en URSS pendant la guerre froide, Thèse de doctorat, Paris, EHESS.

2016 «Les étudiants de pays arabes formés en Union Soviétique pendant la guerre froide », Revue européenne des migrations internationales, 32 (2) : 13-38.

Kernen, A. \& Guex, A.

2016 «Partir étudier en Chine pour faire carrière en Afrique? Les jeunes diplômés africains des universités chinoises expérimentent la localisation du personnel des entreprises chinoises », <https://halshs.archives-ouvertes.fr/halshs$01304697 \mathrm{v} 2>$.

Koudawo, F.

1992 La formation des cadres africains en Europe de l'Est depuis 1918: des nègres rouges aux russotiques, Paris, L'Harmattan. 
Kurgat, P. \& Kurgat, A.

2012 The Shield and the Sickle: Aspects of Kenya-Russian Relations from the Colonial Period to the End of the Cold War (1929-1989), Moscow, РФКИмидж Јаб.

Leclerc-Olive, M. \& Béthune, J.-P.

2013 «Quelques données statistiques sur les flux d'étudiants internationaux. Commentaires et questions », <https://riae.hypotheses.org/207>.

Leclerc-Olive, M.

2016 «Former des élites : mobilités des étudiants d'Afrique au nord du Sahara dans les pays de l'ex-bloc socialiste. Éditorial», Revue européenne des migrations internationales, 32 (2) : 7-12.

MAzov, S.

2015 « Le travail idéologique auprès des étudiants africains dans les établissements d'enseignement supérieur soviétique. Première moitié des années 1960 », in M. de Saint Martin, G. Scarfò Ghellab \& K. Mellakh (dir.), op. cit. : 37-63.

McClellan, W.

2007 "Black Hajj to "Red Mecca": Africans and Afro-Americans at KUTV, 1925-1938 », in M. Matusevitch (ed.), Africa in Russia. Russia in Africa. Three Centuries of Encounters, Trenton N.J.-Amsara, Africa World Press : 61-84.

MellaKh, K.

2016 «La formation des étudiants marocains dans les pays de l'Est de l'Europe (1960-2015) », Revue européenne des migrations internationales, 32 (2) : $39-56$.

Nzula, A. T., Potekhin, I. I. \& Zusmanovich, A. Z.

1984 Forced Labour in Colonial Africa, ed., intro., R. Cohen, trans., H. Jenkins, London, Zed Press.

Potekhin, I. I., Zusmanovich, A. Z. \& Jackson, T.

1933 Prinuditelny trud i profdvizheniie v negritianskoi Afrike (Le mouvement de la classe ouvrière et le travail forcé en Afrique nègre), Moscou, Profizdat.

RIBEIRO, R.

2014 «The Meaning of Internationalism in Angola : Were the Cubans "Exporting" the Revolution or Becoming "the Good Colonizers"? ", Outre-Mers. Revue d'histoire, 102 (384-385) : 267-286.

SADJI, A. B.

2006 Le rôle de la génération charnière ouest-africaine. Indépendance et développement, Paris, L'Harmattan.

de Saint Martin, M., Scarfò Ghellab, G. \& Mellakh, K. (Dir.)

2015 Étudier à l'Est. Expériences de diplômés africains, Préface de J.-P. Dozon, Paris, Karthala-FMSH. 
Simm-Moskovitina, A. \& Dobronravin, N.

2015 «Des élites africaines entre deux mondes. Impact de la formation en URSS ou du poids du milieu social d'origine ? », in M. de SAInt Martin, G. Scarfò Ghellab \& K. Mellakh (dir.), op. cit. : 275-287.

Tchicaya-Oboa, R. \& Yengo, P.

2015 «Être une étudiante africaine en URSS. Sur quelques trajectoires de médecins et pharmaciennes congolaises », in M. de SAint Martin, G. Scarfò Ghellab \& K. Mellakh (dir.), op. cit. : 215-230.

Westad, O. A.

2005 The Global Cold War: Third World Interventions and the Making of Our Times, New York, Cambridge University Press.

WILSON, E. T.

1974 Russia and Black Africa before World War II, New-York, Holmes \& Meier Publishers.

YENGO, P.

2006 La guerre civile du Congo-Brazzaville 1993-2002. Chacun aura sa part, Paris, Karthala.

2011 «Jalons pour une historiographie des élites africaines formées dans les pays de l'Est », in M. Leclerc-Olive, G. Scarfò Ghellab \& A.-C. Wagner (dir.), Les mondes universitaires face au marché. Circulation des savoirs et pratique des acteurs, Paris, Karthala: 135-148. 
